\title{
A singular elliptic equation with natural growth in the gradient and a variable exponent
}

\author{
José Carmona, Pedro J. Martínez-Aparicio and Julio D. Rossi
}

\begin{abstract}
In this paper we consider singular quasilinear elliptic equations with quadratic gradient and a singular term with a variable exponent

$$
\begin{cases}-\Delta u+\frac{|\nabla u|^{2}}{u^{\gamma(x)}}=f & \text { in } \Omega, \\ u=0 & \text { on } \partial \Omega .\end{cases}
$$

Here $\Omega$ is an open bounded set of $\mathbb{R}^{N}, \gamma(x)$ is a positive continuous function and $f$ is positive function that belongs to a certain Lebesgue space. We show, among other results, that there exists a solution in the natural energy space $H_{0}^{1}(\Omega)$ to this problem when $\gamma(x)$ is strictly less than 2 in a strip around the boundary; while there is no solution in the energy space when there exists $\Gamma \subset \partial \Omega$ with $|\Gamma|_{N-1}>0$ such that $\gamma(x)>2$ on $\Gamma$. Moreover, since we work by approximation we can analyze the behavior of the approximated solutions $u_{n}$ in the case in which there is no solution in $H_{0}^{1}(\Omega)$.
\end{abstract}

Mathematics Subject Classification. 35A01, 35B09, 35B45, 35D30, 35J25, 35J60, 35J75.

Keywords. Nonlinear elliptic equations, Singular natural growth gradient terms, Positive solutions, Variable exponent.

\section{Introduction}

In the framework of quasilinear elliptic equations with quadratic growth in the gradient, here we are concerned with the existence of solutions for the following boundary value problem:

$$
\begin{cases}-\Delta u+\frac{|\nabla u|^{2}}{u^{\gamma(x)}}=f & \text { in } \Omega, \\ u=0 & \text { on } \partial \Omega,\end{cases}
$$

where $\Omega$ is an open, bounded subset of $\mathbb{R}^{N}(N \geq 3), 0 \leq f \in L^{q}(\Omega)$ with $q \geq \frac{N}{2}$ satisfying 


$$
m_{\omega}(f) \stackrel{\text { def }}{=} \operatorname{ess} \inf \{f(x): x \in \omega\}>0, \quad \forall \omega \subset \subset \Omega_{\delta}
$$

where $\Omega_{\delta}:=\{x \in \Omega: \operatorname{dist}(x, \partial \Omega)<\delta\}$, for $\delta>0$ fixed, and $\gamma(x) \in C^{1}(\bar{\Omega})$ is a positive function.

If the lower order term is nonsingular, namely

$$
\begin{cases}-\Delta u+g(x, u)|\nabla u|^{2}=f & \text { in } \Omega, \\ u=0 & \text { on } \partial \Omega,\end{cases}
$$

with $g$ a Carathéodory function in $\Omega \times[0, \infty)$, problem (1.3) has been exhaustively studied in $[6,8,12]$ with data $f$ in suitable Lebesgue spaces.

In the case in which the lower order term is singular, there are several papers that deal with existence and nonexistence of solutions when $\gamma$ is a positive constant, namely with the model problem

$$
\begin{cases}-\Delta u+\frac{|\nabla u|^{2}}{u^{\gamma}}=f & \text { in } \Omega, \\ u=0 & \text { on } \partial \Omega .\end{cases}
$$

First, existence of solutions for (1.4) was proved in $[1,3,4]$ for $0<\gamma \leq 1$ and the uniqueness of solution for $0<\gamma<1$ in [5]. We also quote the paper [14]. Specifically, the existence of positive solutions of (1.4) is proved in [9] for $\gamma \leq 1$ provided $0 \not \equiv f \in L^{q}(\Omega)\left(q>\frac{2 N}{N+2}\right)$ with $f \geq 0$. In [2] it is proved the existence of solution if $\gamma<2$ when a strong condition on $f$ is assumed (see [17] for the parabolic case). More precisely, it is imposed condition (1.2) in the whole $\Omega$. Moreover nonexistence is proved if $\gamma>2$ or if $\gamma=2$ and $\lambda_{1}(f)>1$, where $\lambda_{1}(f)$ denotes the first positive eigenvalue of the Laplacian operator $-\Delta$ with zero Dirichlet boundary conditions and weight $f \in L^{q}(\Omega),(q>N / 2)$. In [9] the author proves the same result as in [2] avoiding, in the case $0<\gamma<1$, the assumption that $f$ must be strictly positive in compact subsets of $\Omega$ (see also [16]). Later, in [19] it is proved the nonexistence of solution assuming only that $\gamma \geq 2$.

In the present paper, we deal with a variable exponent and we analyze how the behavior of $\gamma(x)$ influences the existence and nonexistence of solutions. We may have a region inside $\Omega$ where $\gamma(x)<2$ and another region where $\gamma(x) \geq 2$.

The main goal here is to explain that what matters for existence of solutions is the behaviour of $\gamma(x)$ near the boundary.

The idea to prove the existence result consists in approximating the singular term $s^{-\gamma(x)}$ continuously, such that the non singular approximated problems fall into the framework of [13] and therefore they have finite energy solutions $u_{n}$, for every $n \in \mathbb{N}$. We will prove that, for $\gamma(x)<2$ near the boundary, the approximating solutions $u_{n}$ converge to a positive solution of (1.1). As $f \in L^{q}(\Omega)$ with $q \geq \frac{N}{2}$ it is easy to prove ([13]) that exist a priori estimates of the solutions $u_{n}$ in $H_{0}^{1}(\Omega)$. Observe, that due to singularity of the lower order term, the approximated lower order term blows up as $u_{n}(x)$ is converging to zero. This is the reason why it is not possible to apply the ideas of $[6,12,13]$ 
to show the strong convergence of $\nabla u_{n}$ in $L^{2}(\Omega)$ (and thus the strong convergence of the approximated solutions $u_{n}$ in $H_{0}^{1}(\Omega)$ to a solution of (1.1)). The keypoint to overcome this difficulty consists in proving that $u_{n}$ are uniformly away from zero in every compact set inside $\Omega$. We show here that $\gamma(x)$ must be less than 2 only near the boundary for obtaining this kind of estimate. This principle allows us to prove that the sequence of approximating solutions converges locally to a solution of (1.1).

In order to prove our nonexistence result we follow the ideas in [19] adapted for Sobolev functions vanishing only in a part of the boundary.

Our main results are the following (it is assumed that $\partial \Omega$ is Lipschitz and we denote by $n_{e}$ the exterior normal vector to $\partial \Omega$, see the comments before the statement of the main results).

Theorem 1.1. (Existence) Let $f \in L^{q}(\Omega)$ with $q \geq \frac{N}{2}$ satisfying (1.2) and $\gamma(x)<2$ on $\partial \Omega$ or $\gamma(x) \leq 2$ on $\partial \Omega$ with $\frac{\partial \gamma(x)}{\partial n_{e}}>0$, then there exists $u \in$ $H_{0}^{1}(\Omega) \cap L^{\infty}(\Omega)$ a solution to problem (1.1) in the sense of Definition 2.1 below.

Theorem 1.2. (Nonexistence) If there exists $\Gamma \subset \partial \Omega$ with $|\Gamma|_{N-1}>0$ such that $\gamma(x)>2$ on $\Gamma$ or $\gamma(x)=2$ on $\Gamma$ with $\frac{\partial \gamma(x)}{\partial n_{e}} \leq 0$ there then (1.1) admits no solution $u \in H_{0}^{1}(\Omega) \cap L^{\infty}(\Omega)$.

We remark that what we will use to show existence of a solution is that $\gamma(x)<2$ for every $x$ in a strip around $\partial \Omega$ inside $\Omega$. Our assumptions on $\gamma(x)$ in Theorem 1.1 guarantee this fact. Note that we can extend the existence result to functions $\gamma(x)$ such that $\gamma(x)<2$ on $A \subset \partial \Omega$ and $\gamma(x)=2$ on $\partial \Omega \backslash A$ with $\frac{\partial \gamma(x)}{\partial n_{e}}>0$ there.

For the nonexistence part we use that there is an open set $D \subset \Omega$ such that $\gamma(x) \geq 2$ in $D$ and $|\partial D \cap \partial \Omega|_{N-1}>0$. Remark that the conditions on $\gamma(x)$ assumed in Theorem 1.2 imply the existence of such set $D$.

The paper is organized as follows. Section 2 is devoted to describe the approximated problems and we prove some properties that we need in the proof of our main results. In Sect. 3 we prove the main results. We analyze the behavior of the solutions to the approximated problems in Sect. 4 .

Notations. As usual, for every $s \in \mathbb{R}$ we consider the positive and negative parts given by $s^{+}=\max \{s, 0\}$ and $s^{-}=\min \{s, 0\}$. For any $k>0$ we set $T_{k}(s)=\min (k, \max (s,-k))$ and $G_{k}(s)=s-T_{k}(s)$. We denote by $|E|$ the Lebesgue measure of a measurable set $E$ in $\mathbb{R}^{N}$ and by $|\Gamma|_{N-1}$ the $(N-1)$ dimensional surface measure of $\Gamma$. For $1 \leq p \leq+\infty,\|u\|_{p}$ is the usual norm of a function $u \in L^{p}(E)$. We equip the standard Sobolev space $H_{0}^{1}(E)$ with the usual norm $\|u\|=\left(\int_{E}|\nabla u|^{2}\right)^{1 / 2}$.

Moreover, for any $q>1, q^{\prime}=\frac{q}{q-1}$ will be the Hölder conjugate exponent of $q$, while for any $1<p<N, p^{*}=\frac{N p}{N-p}$ is the Sobolev conjugate exponent of $p$. As usual, $\mathcal{S}$ denotes the best Sobolev constant, i.e.,

$$
\mathcal{S}=\sup _{\|u\|_{H_{0}^{1}(\Omega)}=1}\|u\|_{L^{2^{*}(\Omega)}} .
$$


Following [12], we set $\varphi_{\lambda}(s)=s e^{\lambda s^{2}}, \lambda>0$; we will use here that for every $a, b>0$ we have

$$
a \varphi_{\lambda}^{\prime}(s)-b\left|\varphi_{\lambda}(s)\right| \geq \frac{a}{2},
$$

if $\lambda>\frac{b^{2}}{4 a^{2}}$. We will also denote by $\varepsilon(n)$ any quantity that goes to 0 as $n$ goes to infinity.

\section{Preliminary results}

Let us start giving our definition of solution to problem (1.1).

Definition 2.1. We say that $u \in H_{0}^{1}(\Omega) \cap L^{\infty}(\Omega)$ is a positive solution for (1.1) if $u>0$ a.e. $x \in \Omega$,

$$
\frac{|\nabla u|^{2}}{u^{\gamma(x)}} \in L^{1}(\Omega)
$$

and

$$
\int_{\Omega} \nabla u \nabla \varphi+\int_{\Omega} \frac{|\nabla u|^{2}}{u^{\gamma(x)}} \varphi=\int_{\Omega} f(x) \varphi
$$

for every $\varphi \in H_{0}^{1}(\Omega) \cap L^{\infty}(\Omega)$ with $\varphi \geq 0$.

In order to prove our results the approach is to consider the following approximating problems. For every $n \in \mathbb{N}$ let $u_{n}$ be the solution to

$$
\begin{cases}-\Delta u_{n}+\frac{u_{n}^{+}\left|\nabla u_{n}\right|^{2}}{\left(u_{n}^{+}+\frac{1}{n}\right)^{\gamma(x)+1}\left(1+\frac{1}{n}\left|\nabla u_{n}\right|^{2}\right)}=f & \text { in } \Omega, \\ u_{n}=0 & \text { on } \partial \Omega .\end{cases}
$$

Now, we prove some estimates that we will need in what follows.

Proposition 2.2. There exists at least one positive solution $0<u_{n} \in H_{0}^{1}(\Omega) \cap$ $L^{\infty}(\Omega)$ of the approximating problem (2.1). In addition, the sequence $\left\{u_{n}\right\}$ is bounded in $H_{0}^{1}(\Omega)$ and in $L^{\infty}(\Omega)$, i.e. there exists $C>0$ independent of $n$ with

$$
\left\|u_{n}\right\|_{H_{0}^{1}(\Omega)} \leq C,\left\|u_{n}\right\|_{L^{\infty}(\Omega)} \leq C, \quad \forall n \in \mathbb{N} .
$$

Remark 2.3. Standard regularity arguments imply that $u_{n}$ is Hölder continuous.

Proof. Classical results allow us to deduce that the problem (2.1) has a solution $u_{n}$ that belongs to $H_{0}^{1}(\Omega)$ (see $[15]$ ) and to $L^{\infty}(\Omega)$ (see [18]).

To prove the a priori estimate in $L^{\infty}(\Omega)$ we take $\varphi=G_{k}\left(u_{n}\right)$ as test function in (2.1) to obtain

$$
\int_{\Omega}\left|\nabla G_{k}\left(u_{n}\right)\right|^{2}+\int_{\Omega} \frac{u_{n}^{+}\left|\nabla u_{n}\right|^{2}}{\left(u_{n}^{+}+\frac{1}{n}\right)^{\gamma(x)+1}\left(1+\frac{1}{n}\left|\nabla u_{n}\right|^{2}\right)} G_{k}\left(u_{n}\right)=\int_{\Omega} f(x) G_{k}\left(u_{n}\right) .
$$

Using the positivity of the lower order term we deduce that

$$
\int_{\Omega}\left|\nabla G_{k}\left(u_{n}\right)\right|^{2} \leq \int_{\Omega} f(x) G_{k}\left(u_{n}\right) .
$$


Now, by Stampacchia's method, see [18], it follows from this inequality the existence of $C>0$ such that that

$$
\left\|u_{n}\right\|_{L^{\infty}(\Omega)} \leq C .
$$

Now, we prove the a priori estimate in the Sobolev space. Taking $u_{n}$ as test function in (2.1) and using Hölder and Sobolev inequalities we arrive to

$$
\begin{aligned}
& \int_{\Omega}\left|\nabla u_{n}\right|^{2}+\int_{\Omega} \frac{u_{n}^{+}\left|\nabla u_{n}\right|^{2}}{\left(u_{n}^{+}+\frac{1}{n}\right)^{\gamma(x)+1}\left(1+\frac{1}{n}\left|\nabla u_{n}\right|^{2}\right)} u_{n} \\
& \leq\|f\|_{q}\left\|u_{n}\right\|_{q^{\prime}} \leq C \mathcal{S}\|f\|_{q}\left\|u_{n}\right\| .
\end{aligned}
$$

Using the positivity of the lower order term and that $q^{\prime}$ is the conjugate exponent of $q$ (note that for $q>N / 2$ we have $q^{\prime}<2^{*}$ ) we conclude that the sequence $u_{n}$ is bounded in $H_{0}^{1}(\Omega)$. Therefore, up to a subsequence, $u_{n} \rightarrow u$ for some $u \in H_{0}^{1}(\Omega)$.

On the other hand, taking $u_{n}^{-}$as a test function in (2.1) we obtain

$$
\int_{\Omega}\left|\nabla u_{n}^{-}\right|^{2}+\int_{\Omega} \frac{u_{n}^{+}\left|\nabla u_{n}\right|^{2}}{\left(u_{n}^{+}+\frac{1}{n}\right)^{\gamma(x)+1}\left(1+\frac{1}{n}\left|\nabla u_{n}\right|^{2}\right)} u_{n}^{-}=\int_{\Omega} f u_{n}^{-}
$$

and as $f$ is nonnegative we get

$$
\int_{\Omega}\left|\nabla u_{n}^{-}\right|^{2}=\int_{\Omega} f u_{n}^{-} \leq 0 .
$$

Therefore, we deduce that $u_{n} \geq 0$. Moreover, since

$$
-\Delta u_{n}+n^{\|\gamma\|_{L}(\Omega)}+2 u_{n} \geq f
$$

the strong maximum principle assures that $u_{n}>0$.

Now we prove that the solutions of the approximated problems $u_{n}$ are away from zero in every compact subset of $\Omega$. It is in this proof where we appreciate that $\gamma(x)$ must be less than or equal than 2 only near the boundary in order to obtain our existence result.

Proposition 2.4. Let $f \in L^{q}(\Omega)$ with $q \geq \frac{N}{2}$ satisfying (1.2) and $\gamma(x)<2$ on $\partial \Omega$ or $\gamma(x) \leq 2$ on $\partial \Omega$ with $\frac{\partial \gamma(x)}{\partial n_{e}}>0$ then there exists $c_{\omega}>0$ such that $u_{n} \geq c_{\omega}$ for every $\omega \subset \subset \Omega$.

Proof. Let us consider

$$
\Omega_{\eta}=\{x \in \Omega: \operatorname{dist}(x, \partial \Omega)<\eta\} .
$$

Given $\omega \subset \subset \Omega$ there exists $\eta>0$ such that $\omega \subset \Omega \backslash \bar{\Omega}_{\eta}$. The conclusion follows from the fact that there exists $c>0$ such that $u_{n}(x) \geq c$ a.e. $x \in \Omega \backslash \bar{\Omega}_{\eta}$. Note that it is enough to show this for $\eta$ small.

We will prove this fact in two steps. In the first one we prove that there exists $c>0$ such that $u_{n}(x)>c$ for every $x \in \partial\left(\Omega \backslash \bar{\Omega}_{\eta}\right)$. Then, in the second step, we will use this inequality to prove the claim in the whole $\Omega \backslash \bar{\Omega}_{\eta}$. 
Step 1. We may assume that $\eta<\delta$, where $\delta$ is given by (1.2). Since $\gamma(x)<2$ on $\partial \Omega$ or $\gamma(x) \leq 2$ on $\partial \Omega$ with $\frac{\partial \gamma(x)}{\partial n_{e}}>0$ then there exists $\eta_{1} \in(0, \delta)$ such that, for every $\eta<\eta_{1}$ there exists $\gamma_{\eta}^{*}<2$ with

$$
0 \leq \gamma(x) \leq \gamma_{\eta}^{*}<2
$$

for every $x \in \Omega_{\eta} \backslash \bar{\Omega}_{\frac{\eta}{4}}$. Thus we will assume that $0<2 \eta<\eta_{1}<\delta$ and we also have that $\partial\left(\Omega \backslash \bar{\Omega}_{\eta}\right) \subset \omega_{1}$ with

$$
\omega_{1}:=\left\{x \in \Omega: \frac{3 \eta}{4}<\operatorname{dist}(x, \partial \Omega)<\frac{5 \eta}{4}\right\} .
$$

Observe that $\omega_{1} \subset \subset W$ where

$$
W:=\left\{x \in \Omega: \frac{\eta}{2}<\operatorname{dist}(x, \partial \Omega)<2 \eta\right\} \subset \Omega_{2 \eta} \backslash \bar{\Omega}_{\frac{\eta}{2}} .
$$

For every $0<s<C$, with $C$ given by Proposition 2.2, and $x \in W$ we have that

$$
\frac{s}{\left(s+\frac{1}{n}\right)^{\gamma(x)+1}} \leq \frac{(C+1)^{\gamma_{2 \eta}^{*}}}{s^{\gamma_{2 \eta}^{*}}}
$$

Taking

$$
h(s)=\frac{(C+1)^{\gamma_{2 \eta}^{*}}}{s^{\gamma_{2 \eta}^{*}}}
$$

we have that $0<u_{n} \in H^{1}(W) \cap C(W)$ is a supersolution to the equation

$$
-\Delta z+h(z)|\nabla z|^{2}=T_{1}(f) \quad \text { in } W .
$$

Therefore, we can use Proposition 2.3 in [2] (note that condition (1.2) implies that $T_{1}(f)$ satisfies (1.4) of that paper in $W$ and, since $\gamma_{2 \eta}^{*}<2$, the function $h$ satisfies (1.7) of [2]). We deduce the existence of $c_{\omega_{1}}>0$ that $u_{n}(x) \geq c_{\omega_{1}}$ for every $x \in \omega_{1}, n \in \mathbb{N}$.

Step 2. Using that, from Step $1, u_{n}(x) \geq c_{\omega_{1}}$ in $\partial\left(\Omega \backslash \bar{\Omega}_{\eta}\right)$ we prove now that $u_{n} \geq c_{\omega_{1}}$ in $D:=\Omega \backslash \bar{\Omega}_{\eta}$.

We take $\phi_{k} \in C_{0}^{1}(\Omega)$, with $\phi_{k} \geq 0$ and $\operatorname{supp}\left(\phi_{k}\right) \subset \subset D$, as test function in (2.1) and we obtain

$$
\int_{D} \nabla u_{n} \nabla \phi_{k}+\int_{D} \frac{u_{n}\left|\nabla u_{n}\right|^{2}}{\left(u_{n}+\frac{1}{n}\right)^{\gamma(x)+1}\left(1+\frac{1}{n}\left|\nabla u_{n}\right|^{2}\right)} \phi_{k}=\int_{D} f \phi_{k} .
$$

Thus, by density, for every nonnegative $\phi \in H_{0}^{1}(D) \cap L^{\infty}(D)$ we have

$$
\int_{D} \nabla u_{n} \nabla \phi+\int_{D} \frac{u_{n}\left|\nabla u_{n}\right|^{2}}{\left(u_{n}+\frac{1}{n}\right)^{\gamma(x)+1}\left(1+\frac{1}{n}\left|\nabla u_{n}\right|^{2}\right)} \phi=\int_{D} f \phi .
$$

Using that

$$
\frac{u_{n}\left|\nabla u_{n}\right|^{2}}{\left(u_{n}+\frac{1}{n}\right)^{\gamma(x)+1}\left(1+\frac{1}{n}\left|\nabla u_{n}\right|^{2}\right)} \leq(C+1)^{\|\gamma\|_{L} \infty(\Omega)} \frac{\left|\nabla u_{n}\right|^{2}}{\left(u_{n}+\frac{1}{n}\right)^{\|\gamma\|_{L^{\infty}(\Omega)}}}
$$


we obtain, with $c=(C+1)^{\|\gamma\|_{L^{\infty}(\Omega)}}$, that

$$
\int_{D} \nabla u_{n} \nabla \phi+\int_{D} c \frac{\left|\nabla u_{n}\right|^{2}}{\left(u_{n}+\frac{1}{n}\right)^{\|\gamma\|_{L^{\infty}(\Omega)}}} \phi \geq \int_{D} f \phi
$$

for every $0 \leq \phi \in H_{0}^{1}(D) \cap L^{\infty}(D)$.

Now, consider

$$
H_{n}(s)=\int_{1}^{s} \frac{c}{\left(t+\frac{1}{n}\right)^{\|\gamma\|_{L} \infty(\Omega)}} d t
$$

If we take in the previous inequality $e^{-H_{n}\left(u_{n}\right)}\left(c_{\omega_{1}}-u_{n}\right)^{+} \in H_{0}^{1}(D) \cap L^{\infty}(D)$ as test function it follows that

$$
-\int_{D \cap\left\{c_{\omega_{1}} \geq u_{n}\right\}}\left|\nabla u_{n}\right|^{2} e^{-H_{n}\left(u_{n}\right)} \geq \int_{D} f e^{-H_{n}\left(u_{n}\right)}\left(c_{\omega_{1}}-u_{n}\right)^{+} \geq 0 .
$$

Then, $\left(c_{\omega_{1}}-u_{n}\right)^{+} \equiv 0$ and therefore $u_{n} \geq c_{w_{1}}$ in $D$.

\section{Proofs of the main results}

Proof of Theorem 1.1. The result follows from the following steps. First we prove that $u_{n} \rightarrow u$ strongly in $H_{\text {loc }}^{1}(\Omega)$ and next that we can pass to the limit in $(2.1)$.

Step 1. $u_{n} \rightarrow u$ strongly in $H_{\mathrm{loc}}^{1}(\Omega)$. Here we prove that

$$
\lim _{n \rightarrow+\infty} \int_{\Omega}\left|\nabla\left(u_{n}-u\right)\right|^{2} \phi=0, \quad \forall \phi \in C_{0}^{\infty}(\Omega) \text { with } \phi \geq 0 .
$$

Reasoning as in [10], we consider the function $\varphi_{\lambda}(s)$ defined in (1.5) and we choose $\varphi_{\lambda}\left(u_{n}-u\right) \phi$ as test function in (2.1), we have

$$
\begin{aligned}
\int_{\Omega} & \nabla u_{n} \cdot \nabla\left(u_{n}-u\right) \varphi_{\lambda}^{\prime}\left(u_{n}-u\right) \phi+\int_{\Omega} \nabla u_{n} \cdot \nabla \phi \varphi_{\lambda}\left(u_{n}-u\right) \\
& +\int_{\Omega} \frac{u_{n}\left|\nabla u_{n}\right|^{2}}{\left(u_{n}+\frac{1}{n}\right)^{\gamma(x)+1}\left(1+\frac{1}{n}\left|\nabla u_{n}\right|^{2}\right)} \varphi_{\lambda}\left(u_{n}-u\right) \phi \\
& =\int_{\Omega} f \varphi_{\lambda}\left(u_{n}-u\right) \phi .
\end{aligned}
$$

Since, up to a subsequence, $u_{n} \rightarrow u$ weakly in $H_{0}^{1}(\Omega)$ and strongly in $L^{2}(\Omega)$, we note that

$$
\int_{\Omega} f \varphi_{\lambda}\left(u_{n}-u\right) \phi-\int_{\Omega} \nabla u_{n} \cdot \nabla \phi \varphi_{\lambda}\left(u_{n}-u\right)=\varepsilon(n) .
$$

Moreover, choosing $\omega_{\phi} \subset \subset \Omega$ with supp $\phi \subset \omega_{\phi}$, from Propositions 2.2, 2.4 and the fact that $\gamma(x) \in C(\bar{\Omega})$, we deduce that

$$
\begin{gathered}
\int_{\Omega} \frac{u_{n}\left|\nabla u_{n}\right|^{2}}{\left(u_{n}+\frac{1}{n}\right)^{\gamma(x)+1}\left(1+\frac{1}{n}\left|\nabla u_{n}\right|^{2}\right)} \varphi_{\lambda}\left(u_{n}-u\right) \phi \\
\geq-c\left(\omega_{\phi}\right) \int_{\Omega}\left|\nabla u_{n}\right|^{2}\left|\varphi_{\lambda}\left(u_{n}-u\right)\right| \phi .
\end{gathered}
$$


Thus, it follows that

$$
\int_{\Omega} \nabla u_{n} \cdot \nabla\left(u_{n}-u\right) \varphi_{\lambda}^{\prime}\left(u_{n}-u\right) \phi-c\left(\omega_{\phi}\right) \int_{\Omega}\left|\nabla u_{n}\right|^{2}\left|\varphi_{\lambda}\left(u_{n}-u\right)\right| \phi \leq \varepsilon(n) .
$$

Adding

$$
-\int_{\Omega} \nabla u \cdot \nabla\left(u_{n}-u\right) \varphi_{\lambda}^{\prime}\left(u_{n}-u\right) \phi=\varepsilon(n)
$$

to both sides of (3.2) and since

$$
\begin{aligned}
\int_{\Omega}\left|\nabla u_{n}\right|^{2}\left|\varphi_{\lambda}\left(u_{n}-u\right)\right| \phi \leq & 2 \int_{\Omega}\left|\nabla\left(u_{n}-u\right)\right|^{2}\left|\varphi_{\lambda}\left(u_{n}-u\right)\right| \phi \\
& +2 \int_{\Omega}|\nabla u|^{2}\left|\varphi_{\lambda}\left(u_{n}-u\right)\right| \phi \\
= & 2 \int_{\Omega}\left|\nabla\left(u_{n}-u\right)\right|^{2}\left|\varphi_{\lambda}\left(u_{n}-u\right)\right| \phi+\varepsilon(n),
\end{aligned}
$$

we find

$$
\int_{\Omega}\left|\nabla\left(u_{n}-u\right)\right|^{2}\left[\varphi_{\lambda}^{\prime}\left(u_{n}-u\right)-2 c\left(\omega_{\phi}\right)\left|\varphi_{\lambda}\left(u_{n}-u\right)\right|\right] \phi \leq \varepsilon(n) .
$$

Choosing $\lambda$ such that (1.5) holds with $a=1$ and $b=2 c\left(\omega_{\phi}\right)$, we conclude that (3.1) is satisfied.

Step 2. We pass to the limit in (2.1). Choosing $\frac{1}{\varepsilon} T_{\varepsilon}\left(u_{n}\right)$ as test function in (2.1), we obtain

$$
\int_{\Omega} \frac{T_{\varepsilon}\left(u_{n}\right)}{\varepsilon} \frac{u_{n}\left|\nabla u_{n}\right|^{2}}{\left(u_{n}+\frac{1}{n}\right)^{\gamma(x)+1}\left(1+\frac{1}{n}\left|\nabla u_{n}\right|^{2}\right)} \leq \int_{\Omega} f .
$$

If we take the limit as $\varepsilon$ tends to zero, and we use that $u_{n}>0$ in $\Omega$, we get

$$
\int_{\Omega} \frac{u_{n}\left|\nabla u_{n}\right|^{2}}{\left(u_{n}+\frac{1}{n}\right)^{\gamma(x)+1}\left(1+\frac{1}{n}\left|\nabla u_{n}\right|^{2}\right)} \leq \int_{\Omega} f
$$

Since

$$
-\Delta u_{n}=f-\frac{u_{n}\left|\nabla u_{n}\right|^{2}}{\left(u_{n}+\frac{1}{n}\right)^{\gamma(x)+1}\left(1+\frac{1}{n}\left|\nabla u_{n}\right|^{2}\right)},
$$

and the right hand side is bounded in $L^{1}(\Omega)$ by the assumptions on $f$ and by (3.3). Then we can apply Lemma 1 of [7] (see also [11]) to deduce that, up to (not relabeled) subsequences, $\nabla u_{n}$ converges to $\nabla u$ a.e. in $\Omega$.

Using Fatou lemma in (3.3), we get

$$
\int_{\Omega} \frac{|\nabla u|^{2}}{u^{\gamma(x)}} \leq \int_{\Omega} f
$$

Therefore, to conclude the proof we only have to show that $u$ is a distributional solution of the problem (2.1). We begin by passing to the limit as $n \rightarrow \infty$ in the equation satisfied by $u_{n}$, that is, in 


$$
\int_{\Omega} \nabla u_{n} \cdot \nabla \phi+\int_{\Omega} \frac{u_{n}\left|\nabla u_{n}\right|^{2}}{\left(u_{n}+\frac{1}{n}\right)^{\gamma(x)+1}\left(1+\frac{1}{n}\left|\nabla u_{n}\right|^{2}\right)} \phi=\int_{\Omega} f \phi, \forall \phi \in C_{0}^{\infty}(\Omega) .
$$

First of all, the weak convergence of $u_{n}$ to $u$ implies that

$$
\lim _{n \rightarrow+\infty} \int_{\Omega} \nabla u_{n} \nabla \phi=\int_{\Omega} \nabla u \nabla \phi, \quad \forall \phi \in C_{0}^{\infty}(\Omega) .
$$

On the other hand, if we fix $\omega \subset \subset \Omega$, then, by Propositions 2.2, 2.4 and since $\gamma(x) \in C(\bar{\Omega})$, we get

$$
\frac{u_{n}}{\left(u_{n}+\frac{1}{n}\right)^{\gamma(x)+1}} \leq c(\omega), \quad \forall n>>1, \quad \text { and } \forall x \in \omega .
$$

Consequently, if $E \subset \omega$ it follows that

$$
\int_{E} \frac{u_{n}\left|\nabla u_{n}\right|^{2}}{\left(u_{n}+\frac{1}{n}\right)^{\gamma(x)+1}\left(1+\frac{1}{n}\left|\nabla u_{n}\right|^{2}\right)} \leq c(\omega) \int_{E}\left|\nabla u_{n}\right|^{2} .
$$

Let $\varepsilon>0$ be fixed. Since $u_{n}$ is strongly compact in $H_{\text {loc }}^{1}(\Omega)$ and there exist $n_{\varepsilon}, \delta_{\varepsilon}$ such that for every $E \subset \omega \subset \subset \Omega$ with meas $(E)<\delta_{\varepsilon}$, we have

$$
\int_{E}\left|\nabla u_{n}\right|^{2}<\frac{\varepsilon}{c(\omega)}, \quad \forall n \geq n_{\varepsilon} .
$$

In conclusion, by $(3.5)$, we see that meas $(E)<\delta_{\varepsilon}$ implies

$$
\int_{E} \frac{u_{n}\left|\nabla u_{n}\right|^{2}}{\left(u_{n}+\frac{1}{n}\right)^{\gamma(x)+1}\left(1+\frac{1}{n}\left|\nabla u_{n}\right|^{2}\right)} \leq \varepsilon, \quad \forall n \geq n_{\varepsilon}
$$

i.e., the sequence

$$
\frac{u_{n}\left|\nabla u_{n}\right|^{2}}{\left(u_{n}+\frac{1}{n}\right)^{\gamma(x)+1}\left(1+\frac{1}{n}\left|\nabla u_{n}\right|^{2}\right)}
$$

is equiintegrable. This, together with its a.e. convergence to $\frac{|\nabla u|^{2}}{u^{\gamma(x)}}$, implies by Vitali's theorem that

$$
\lim _{n \rightarrow+\infty} \int_{\Omega} \frac{u_{n}\left|\nabla u_{n}\right|^{2}}{\left(u_{n}+\frac{1}{n}\right)^{\gamma(x)+1}\left(1+\frac{1}{n}\left|\nabla u_{n}\right|^{2}\right)} \phi=\int_{\Omega} \frac{|\nabla u|^{2}}{u^{\gamma(x)}} \phi, \quad \forall \phi \in C_{0}^{\infty}(\Omega) .
$$

Therefore, using the above limit and (3.4) we conclude that

$$
\int_{\Omega} \nabla u \nabla \phi+\int_{\Omega} \frac{|\nabla u|^{2}}{u^{\gamma(x)}} \phi=\int_{\Omega} f \phi, \quad \forall \phi \in C_{0}^{\infty}(\Omega),
$$

as we wanted to show.

Now we prove our nonexistence result.

Proof of Theorem 1.2. From our hypothesis, we may assume that $\Gamma=\partial D \cap \partial \Omega$ with $D \subset \Omega$ open such that $\gamma(x) \geq 2$ for every $x \in D$. 
We prove the result using the ideas of [19]. Assume on the contrary that there exists some $u \in H_{0}^{1}(\Omega) \cap L^{\infty}(\Omega)$ solution of (1.1) with $u>0$ a.e. in $\Omega$ such that

$$
\int_{\Omega} \frac{|\nabla u|^{2}}{u^{\gamma(x)}} d x<+\infty .
$$

Since $\gamma(x) \geq 2$, we know in $D$ that

$$
\int_{D} \frac{|\nabla u|^{2}}{(u+\varepsilon)^{2}} \leq \int_{D} \frac{|\nabla u|^{2}}{u^{2}} \leq c \int_{D} \frac{|\nabla u|^{2}}{u^{\gamma(x)}}<+\infty, \quad \forall \varepsilon>0,
$$

i.e.,

$$
\int_{D}|\nabla(\ln (u+\varepsilon)-\ln (\varepsilon))|^{2} \leq C_{3}, \quad \forall \varepsilon>0 .
$$

Denoting $z_{\varepsilon}=|\ln (u+\varepsilon)-\ln (\varepsilon)|$, we have that $z_{\varepsilon} \in H^{1}(D)$ with $z_{\varepsilon}=0$ on $\partial D \cap \partial \Omega$. Now we observe that there exists a constant $C_{4}$ such that

$$
\int_{D} g^{2} \leq C_{4} \int_{D}|\nabla g|^{2}
$$

for any function $g \in H^{1}(D)$ with $g=0$ on $\Gamma$. To see this fact, we argue by contradiction. Assume that there is a sequence $g_{n}$ such that $\int_{D}\left|\nabla g_{n}\right|^{2} \rightarrow 0$ and $\int_{D} g_{n}^{2}=1$. Then $g_{n}$ converges strongly in $H^{1}(D)$ to a function $g_{0}$ that verifies $\int_{D}\left|\nabla g_{0}\right|^{2}=0$ (hence, $g_{0}$ is a constant) $\int_{D} g_{0}^{2}=1$ and $g_{0}=0$ on $\Gamma$, a contradiction. Thus, using the generalized Poincaré's inequality (3.6) we get

$$
\int_{D} z_{\varepsilon}^{2} \leq C_{4} \int_{D}\left|\nabla z_{\varepsilon}\right|^{2} \leq C_{4} C_{3}:=C_{5}, \quad \forall \varepsilon>0 .
$$

Denote $E_{n}=\left\{x \in D: u(x)>\frac{1}{n}\right\}$ for every $n \in \mathbb{N}$. Then we have

$$
\{x \in D ; u(x)>0\}=\bigcup_{n=1}^{\infty} E_{n},
$$

which implies that

$$
0<|D| \leq \sum_{n=1}^{\infty}\left|E_{n}\right|
$$

and then there exists $n_{0} \in \mathbb{N}$ such that $\left|E_{n_{0}}\right|>0$. We deduce

$$
\left|\ln \left(\frac{1}{n_{0}}+\varepsilon\right)-\ln (\varepsilon)\right|^{2} \cdot\left|E_{n_{0}}\right| \leq \int_{E_{n_{0}}}|\ln (u+\varepsilon)-\ln (\varepsilon)|^{2} \leq C_{5},
$$

for every $\varepsilon>0$, therefore

$$
\left|\ln \left(\frac{1}{n_{0}}+\varepsilon\right)-\ln (\varepsilon)\right|^{2} \leq \frac{C_{5}}{\left|E_{n_{0}}\right|}<+\infty, \quad \forall \varepsilon>0 .
$$

Now, as $\varepsilon$ goes to zero, we obtain a contradiction. 


\section{Behavior of the approximating solutions $\boldsymbol{u}_{n}$}

In this section we analyze the behavior of the solutions of the approximating problems (2.1) in the case in which there is no solution in the Sobolev space $H_{0}^{1}(\Omega)$.

We consider here the case $\bar{\Omega}=\bar{D}_{1} \cup \bar{D}_{2}$, where $D_{1}, D_{2} \subset \Omega$ are open sets with $\left|\partial D_{2} \cap \partial \Omega\right|_{N-1}>0$ and

$$
\begin{array}{ll}
\gamma(x)<2 & \text { for every } x \in D_{1}, \\
\gamma(x) \geq 2 & \text { for every } x \in \bar{D}_{2} .
\end{array}
$$

This will be referred as condition $(H)$.

In this case Theorem 1.2 assures that there is no solution $u \in H_{0}^{1}(\Omega) \cap$ $L^{\infty}(\Omega)$ of (1.1). We explain what occurs with the approximations $u_{n}$ in the following result.

Theorem 4.1. Assume (1.2) for every $\delta>0$ and that condition $(H)$ is satisfied. Then the weak limit $u$ of the sequence $u_{n}$ satisfies that $0<u$ in $D_{1}, u \equiv 0$ in $D_{2}, u \in H_{0}^{1}\left(D_{1}\right) \cap L^{\infty}(\Omega)$ and $u$ satisfies

$$
\begin{cases}-\Delta u+\frac{|\nabla u|^{2}}{u^{\gamma(x)}}=f & \text { in } D_{1} \\ u=0 & \text { on } \partial D_{1} .\end{cases}
$$

Moreover, there exists a Radon measure $\nu_{0} \in \mathcal{M}(\Omega)$ supported in $D_{2}$ such that, in the sense of distributions,

$$
\begin{cases}-\Delta u+\frac{|\nabla u|^{2}}{u^{\gamma(x)}} \chi_{D_{1}}=f-\nu_{0} & \text { in } \Omega \\ u=0 & \text { on } \partial \Omega\end{cases}
$$

Proof. Observe that the sequence $u_{n}$ of solutions of (2.1) weakly converges in $H_{0}^{1}(\Omega)$ to $u \in H_{0}^{1}(\Omega) \cap L^{\infty}(\Omega)$ (using the Sobolev's estimate proved in Proposition 2.2). Moreover, Proposition 2.4 is valid for $\omega \subset \subset D_{1}$ (observe that what we use in that result is that $\gamma(x)<2$ for every $x$ in a strip around $\partial \Omega$ inside $\Omega$ ) and, in particular, $u>0$ in $D_{1}$. Even more, as in (3.3) we have that

$$
\nu_{n}=\frac{u_{n}\left|\nabla u_{n}\right|^{2}}{\left(u_{n}+\frac{1}{n}\right)^{\gamma(x)+1}\left(1+\frac{1}{n}\left|\nabla u_{n}\right|^{2}\right)} \quad \text { is bounded in } L^{1}(\Omega),
$$

therefore, the result of [11] yields that (up to subsequences) $\nabla u_{n}$ converges to $\nabla u$ almost everywhere in $\Omega$. Thus there exists a positive Radon measure $\nu \in \mathcal{M}(\Omega)$ such that, up to a subsequence, $\nu_{n} \rightarrow \nu$ in the weak-* topology of measures. Since we can use Fatou lemma to obtain that $\frac{|\nabla u|^{2}}{u^{\gamma(x)}} \in L^{1}\left(D_{1}\right)$ we can even assume that $\nu=\frac{|\nabla u|^{2}}{u^{\gamma(x)}} \chi_{D_{1}}(x)+\nu_{0}$, where $\nu_{0}$ is a nonnegative bounded Radon measure on $\Omega$.

Now we claim that $u=0$ in $\bar{D}_{2}$. Indeed, if $D=\left\{x \in \bar{D}_{2}: u(x)>0\right\}$ and $|D|>0$ then, since $\gamma(x) \geq 2$ in $\bar{D}_{2}$ we can argue as in the proof of Theorem 1.2 
(observe that $u \in H^{1}(D)$ and $u=0$ on a subset of $\partial D$ of positive measure). For example, if $D=D_{2}$ then $u=0$ on $\partial D_{2} \cap \partial \Omega$. As another example, we mention that if $\bar{D} \subset D_{2}$ then $u=0$ on $\partial D$. Thus we reach a contradiction and the claim is proved.

As a consequence $u \in H_{0}^{1}\left(D_{1}\right)$ and, as in the proof of Theorem 1.1, we can pass to the limit in the approximating problems to prove (4.1). In addition, (4.2) follows from the weak-* convergence of $\nu_{n}$. Finally, in order to prove that $\nu_{0}$ is supported in $D_{2}$ we observe that, taking $\phi \in C_{0}^{\infty}\left(D_{1}\right)$ as test function in (4.2) and (4.1) and subtracting we obtain that

$$
\int_{\Omega} \phi d \nu_{0}=0
$$

On the other hand, taking $\phi \in C_{0}^{\infty}\left(D_{2}\right)$ as test function in (4.2) and using that $u=0$ in $D_{2}$ we get that

$$
\int_{\Omega} \phi d \nu_{0}=\int_{\Omega} f \phi
$$

Remark 4.2. We remark that when we consider $(H)$ in the case $\partial D_{2} \cap \partial \Omega=\emptyset$ we have proved that the weak limit $u$ of the sequence $u_{n}$ satisfies that $0<u$ in $\Omega$ and it is a solution to (1.1). This is a consequence of the fact that we have $\gamma(x)<2$ in a strip near the boundary of $\Omega$, and hence the approximations converge to a solution to (1.1) as was proved in Theorem 1.1.

The case in which $\partial D_{2} \cap \partial \Omega \neq \emptyset$ with $\left|\partial D_{2} \cap \partial \Omega\right|_{N-1}=0$ is left open.

Remark 4.3. Finally we point out that, as in [2] or [9], the above results can be generalized to a more general class of differential operators. More precisely we can consider

$$
\begin{cases}-\operatorname{div}(M(x, u) \nabla u)+Q(x, u) \frac{|\nabla u|^{2}}{u^{\gamma(x)}}=f & \text { in } \Omega, \\ u=0 & \text { on } \partial \Omega,\end{cases}
$$

with $M(x, s)$ a matrix with coefficients $m_{i, j}(x, s)$, such that $Q$ and $m_{i, j}$ are Carathéodory functions, $i, j=1, \ldots, N$ and for some positive constants $a, b$, $\alpha, \beta$ it is satisfied that

$$
\begin{gathered}
0<a \leq Q(x, s) \leq b, \quad s>0 \\
0<\alpha|\xi|^{2} \leq M(x, s) \xi \cdot \xi, \quad|M(x, s)| \leq \beta, \quad s>0, x \in \Omega, \quad \xi \in \mathbb{R}^{N} .
\end{gathered}
$$

\section{Acknowledgments}

Research supported by MICINN Ministerio de Ciencia e Innovación, Spain under grant MTM2012-31799 and Junta de Andalucía FQM-194 (J. Carmona) and FQM-116 (P. J. Martínez-Aparicio). 


\section{References}

[1] Arcoya, D., Barile, S., Martínez-Aparicio, P.J.: Singular quasilinear equations with quadratic growth in the gradient without sign condition. J. Math. Anal. Appl. 350, 401-408 (2009)

[2] Arcoya, D., Carmona, J., Leonori, T., Martínez-Aparicio, P.J., Orsina, L., Petitta, F.: Existence and nonexistence of solutions for singular quadratic quasilinear equations. J. Differ. Equ. 246, 4006-4042 (2009)

[3] Arcoya, D., Carmona, J., Martínez-Aparicio, P.J.: Elliptic obstacle problems with natural growth on the gradient and singular nonlinear terms. Adv. Nonlinear Stud. 7, 299-317 (2007)

[4] Arcoya, D., Martínez-Aparicio, P.J.: Quasilinear equations with natural growth. Rev. Mat. Iberoam. 24, 597-616 (2008)

[5] Arcoya, D., Segura de León, S.: Uniqueness of solutions for some elliptic equations with a quadratic gradient term. ESAIM Control Optim. Calc. Var. 10(2), 327-336 (2010)

[6] Bensoussan, A., Boccardo, L., Murat, F.: On a nonlinear P.D.E. having natural growth terms and unbounded solutions. Ann. Inst. Henri Poincaré Anal. Non Linéaire 5, 347-364 (1988)

[7] Boccardo, L., Gallouët, T.: Nonlinear elliptic and parabolic equations involving measure data. J. Funct. Anal. 87, 149-169 (1989)

[8] Boccardo, L., Gallouët, T.: Strongly nonlinear elliptic equations having natural growth terms and $L^{1}$ data. Nonlinear Anal. 19, 573-579 (1992)

[9] Boccardo, L.: Problems with singular and quadratic gradient lower order terms. ESAIM Control Optim. Calc. Var. 14, 411-426 (2008)

[10] Boccardo, L., Gallouët, T., Murat, F.: A unified presentation of two existence results for problems with natural growth. In: Progress in Partial Differential Equations: The Metz Surveys, vol. 2, pp. 127-137 (1992) (Pitman Research Notes in Mathematics Series, vol. 296, Longman Science and Technology, Harlow, 1993)

[11] Boccardo, L., Murat, F.: Almost everywhere convergence of the gradients of solutions to elliptic and parabolic equations. Nonlinear Anal. 19, 581-597 (1992)

[12] Boccardo, L., Murat, F., Puel, J.-P.: Existence de solutions non bornees pour certaines équations quasi-linéaires. Portugaliae Math. 41, 507-534 (1982)

[13] Boccardo, L., Murat, F., Puel, J.-P.: $L^{\infty}$ estimate for some nonlinear elliptic partial differential equations and application to an existence result. SIAM J. Math. Anal. 23, 326-333 (1992)

[14] Giachetti, D., Murat, F.: An elliptic problem with a lower order term having singular behaviour. Boll. Unione Mat. Ital. (9) 2(2), 349-370 (2009) 
[15] Leray, J., Lions, J.L.: Quelques résultats de Višik sur les problèmes elliptiques non linéaires par les méthodes de Minty-Browder. Bull. Soc. Math. France 93, 97-107 (1965)

[16] Martínez-Aparicio, P.J.: Dirichlet problems with quadratic gradient. Boll. Unione Mat. Ital. (9) 2(3), 559-574 (2009)

[17] Martínez-Aparicio, P.J., Petitta, F.: Parabolic equations with nonlinear singularities. Nonlinear Anal. 74(1), 114-131 (2011)

[18] Stampacchia, G.: Equations Èlliptiques du Second Ordre à Coefficients Discontinus, vol. 35.45, p. 326. Les Presses de l'Université de Montréal, Montreal (1966)

[19] Zhou, W., Wei, X., Qin, X.: Nonexistence of solutions for singular elliptic equations with a quadratic gradient term. Nonlinear Anal. 75, 5845-5850 (2012)

José Carmona

Departamento de Matemáticas

Universidad de Almería

Ctra. Sacramento s/n, La Cañada de San Urbano

04120 Almería

Spain

e-mail: jcarmona@ual.es

Pedro J. Martínez-Aparicio

Departamento de Matemática Aplicada y Estadística

Campus Alfonso XIII

Universidad Politécnica de Cartagena

30203 Murcia

Spain

e-mail: pedroj.martinez@upct.es

Julio D. Rossi

Departamento de Matemática, FCEyN UBA

Ciudad Universitaria

Pab 1, 1428 Buenos Aires

Argentina

e-mail: jrossi@dm.uba.ar

Received: 14 May 2015.

Accepted: 22 August 2015. 